\title{
ASPECTOS ECOTOXICOLÓGICOS DE HIDROCARBONETOS POLICÍCLICOS AROMÁTICOS
}

\author{
Rodrigo Ornellas Meire ${ }^{1 *}$, Antonio Azeredo ${ }^{1,2} \&$ João Paulo Machado Torres ${ }^{1}$ \\ ${ }^{1}$ Laboratório de Radioisótopos Eduardo Penna Franca, Instituto de Biofísica Carlos Chagas Filho. Universidade Federal do Rio de Janeiro, Prédio do \\ Centro de Ciências e Saúde - Bloco G, Sala 62 - Subsolo. Ilha do Fundão, Cidade Universitária, CEP: 21941-902 - Rio de Janeiro, Brasil. ²Laboratório \\ de Toxicologia, Faculdade de Ciências Farmacêuticas - Departamento de Saúde. Universidade Estadual de Feira de Santana, Av. Universitária s/n - Km \\ 03, BR 116 Norte, CEP.: 44031-160 - Feira de Santana - Bahia, Brasil. \\ *E-mail: romeire@biof.ufrj.br
}

\section{RESUMO}

Hidrocarbonetos Policíclicos Aromáticos (HPAs) são considerados poluentes orgânicos prioritários em estudos ambientais. Algumas dessas substâncias podem ser consideradas precursoras de ações mutagênicas e tumorais em sistemas biológicos. HPAs são formados exclusivamente por átomos de carbono e hidrogênio e organizados sob a forma de anéis aromáticos fusionados entre si. Essa classe de substâncias tem sua origem na combustão incompleta da matéria orgânica regida principalmente por diferentes fatores físicos, como temperatura e pressão. O transporte de HPAs se dá principalmente através de material particulado fino atmosférico ou por meio aquoso, podendo atingir desta forma regiões distantes de suas origens. HPAs são altamente lipossolúveis e rapidamente absorvidos pelos pulmões, intestinos e pele de homens e animais. Uma vez absorvidos pelas células, os HPAs são metabolicamente ativados e, desta maneira, tornam-se reativos a grupos nuclofílicos presentes em macromoléculas celulares. A formação de adutos de DNA é considerada essencial na carcinogenicidade química desses xenobiontes. Consumidores de alimentos defumados, fumantes, indivíduos em contato com ambiente contaminado (i.e. água e ar), e trabalhadores ocupacionais expostos de forma direta a HPAs podem apresentar elevadas propensões ao desenvolvimento de tumores e câncer.

Palavras-chave: HPA, benzo(a)pireno, ecotoxicologia, poluição, carcinogênico.

\section{ABSTRACT}

ECOTOXICOLOGY ASPECTS FROM POLICICLIC AROMATIC HYDROCARBONS (PAH). Policiclic Aromatic Hydrocarbons (PAH) are considered priority organic pollutants in environmental researches. Some of these substances can be considered mutagenic and tumors precursors in biologic systems. $\mathrm{PAH}$ are formed exclusively by carbon and hydrogen atoms consisting of two or more fused aromatic rings arrangements. PAH are originated from incomplete burning of organic matter leading by different physics factors as temperature and pressure parameters. $\mathrm{PAH}$ are transported mainly through fine particles in atmosphere or aquatic environment and can reach distant areas far from its origins. PAH are lipophilic compounds and quickly absorbed by lung, intestine and skin from human and animals. Once absorbed by cells, PAH are metabolic activated and this way becomes reactive to nucleophilic groups of cellular macromolecules. The formation of DNA adducts is considered essential in the chemical carcinogenic of these xenobiotic compounds. The consummers of smoked foods, people that smokes, live in contaminated environment (e.g. water and air), and occupational workers who are exposed in a direct form to PAH present the higher risks of tumors and cancer development.

Key-words: PAH, benze(a)pyrene, ecotoxicology, pollution, carcinogenic.

\section{CARACTERÍSTICAS GERAIS}

Hidrocarbonetos Policíclicos Aromáticos (HPAs) são considerados poluentes orgânicos prioritários em estudos ambientais, sendo alguns desses contaminantes descritos como precursores de ações mu- tagênicas e tumorais em sistemas biológicos (WHO 1983). HPAs são compostos aromáticos formados por dois ou mais anéis benzênicos, constituídos exclusivamente por átomos de carbono e hidrogênio (Figura 1), organizados sob forma linear, angular ou agrupada (Netto et al. 2000). A formação desses 
contaminantes tem sua origem na combustão incompleta da matéria orgânica, origem essa influenciada principalmente por fatores como temperatura e pressão que direcionam o perfil constituinte dos mesmos (Page et al. 1999). Deste modo, incêndios florestais e de campos, assim como a queima de combustível fóssil, seriam as principais fontes de HPAs para o meio ambiente. Porém, as maiores emissões provêm de processos industriais ligados à produção de aço e alumínio, da exaustão de incineradores de rejeito e por resíduos sólidos industriais. Atividades petroquímicas como o processo e refino na produção de petróleo, assim como, acidentes que envolvem o derramamento direto de seus produtos e derivados em corpos receptores, como lagos, rios e oceanos, também elevam consideravelmente os níveis ambientais de HPAs (Page et al. 1999, Yunker et al. 2002, Sisinno et al. 2003).

De acordo com a agência de proteção ambiental dos Estados Unidos (EPA - Environment Protection Agency) 16 hidrocarbonetos poliaromáticos são con- siderados particularmente importantes no monitoramento ambiental de poluentes orgânicos prioritários (EPA 1986): Acenafteno acenaftileno, antraceno, benzo(a)antraceno, benzo(a)pireno, benzo(b)fluoranteno, benzo(ghi)perileno, benzo(k)fluoranteno, criseno, dibenzo(a,h)antraceno, fenantreno, fluoranteno, fluoreno, indeno(1,2,3-cd)pireno, naftaleno e pireno (Figura 1). Esses compostos apresentam de 2 a 6 anéis aromáticos fundidos entre si com peso molecular (PM) variando entre 128 e $278 \mathrm{~g} / \mathrm{mol}$. Suas características físico-químicas, como solubilidade $(S)$ e pressão de vapor $(P V)$, são fatores importantes que direcionam a distribuição desses contaminantes entre as fases solúvel e particulada em meio atmosférico, aquoso e biótico (Tabela 1). A solubilidade em água dos HPAs varia entre os altamente insolúveis (e.g. benzo[g,h,i]perileno: 0,003mg/L) a pouco solúveis em água (e.g. naftaleno, 31mg/L), enquanto a pressão de vapor transita entre compostos altamente voláteis (naftaleno) e compostos relativamente pouco voláteis (dibenzo[a,h]antraceno).

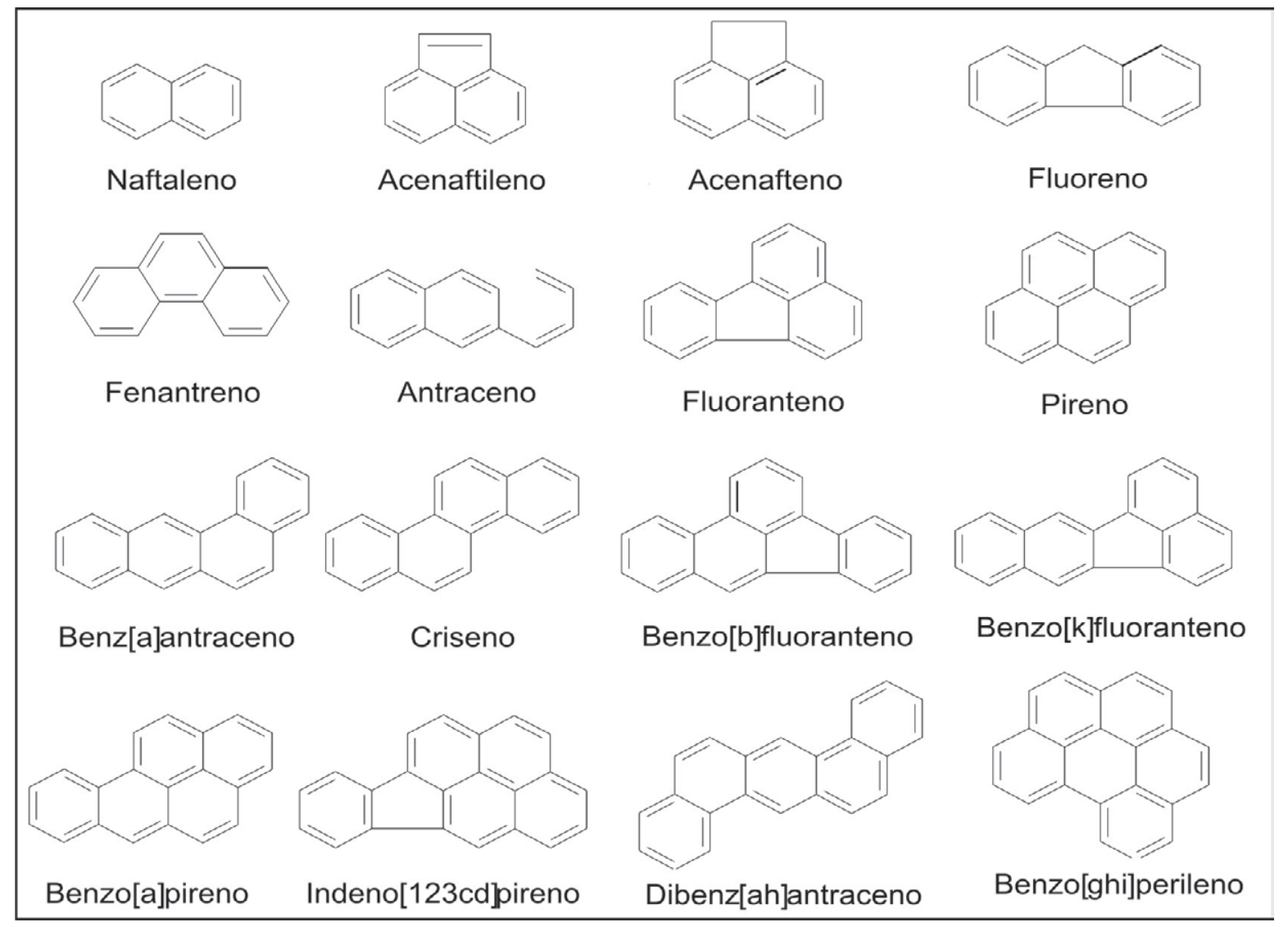

Figura 1. Hidrocarbonetos Policíclicos Aromáticos (HPAs). Nesta figura estão os 16 HPAs prioritários em estudos ambientais de acordo com a Agência de Proteção Ambiental dos Estados Unidos (EPA 1987). 
O transporte desses poluentes no ambiente se dá principalmente por via atmosférica associado ao material particulado fino, o que permite uma ampla distribuição desses compostos no ambiente. Uma vez emitidos na atmosfera, os HPAs podem ser depositados sob a forma seca (vapor ou particulada) ou úmida (precipitação sob a forma dissolvida ou particulada) sobre sistemas aquáticos e terrestres (Garban et al. 2002, Rose \& Rippey 2002). O comportamento de partição entre água e ar, entre água e sedimento e entre água e a biota são características importantes na distribuição de HPAs no ambiente. Esses poluentes apresentam alto coeficiente de partição entre solventes orgânicos e água, o que nos permite prever possíveis processos cumulativos em compartimentos como sedimentos e solos (ricos em matéria orgânica adsorvida), assim como na bioacumulação em sistemas biológicos (Neff 1984).

Como pode ser observado na Tabela 1 , o peso molecular está inversamente relacionado com a solubilidade dos HPAs que diminui com o aumento do número de anéis. Os HPAs apresentam características lipofílicas, onde seus constituintes são classificados como moderadamente a altamente lipossolúveis, apresentando coeficientes de partição octanol-água $\left(\log \mathrm{K}_{\mathrm{oa}}\right.$ ) que variam entre 3,37 e 6,75. O mesmo pode ser relacionado com a volatilidade dessa classe de substâncias, onde compostos com menores pesos moleculares apresentam elevada pressão de vapor e conseqüentemente ampla dispersão ambiental (Netto et al. 2000, Brito et al. 2005).

A degradação ambiental de HPAs pode se dá por processos físico-químicos, como no caso da degradação por fotooxidação, ou pela ação biológica de microorganismos (Hwang \& Cutright 2002). Muitos estudos vêm demonstrando a capacidade hidrocarbonoclastica de diferentes espécies de bactérias, fungos e leveduras (Hedlund et al. 2001, Chaillan et al. 2004). Essa capacidade nos permite observar o potencial papel biotecnológico dessas espécies na biorremediação de ambientes contaminados (Brito et al. 2006). HPAs também podem ser biotransformados pela ação de enzimas específicas, muitas vezes presentes em grandes vertebrados como no caso de mamíferos e aves (Warshawsky 1999). O processo de biotransformação dos HPAs é crucial na ativação e formação de possíveis agentes carcinogênicos (Hall et al. 1989). As propriedades mutagênicas e carcinogênicas de alguns HPAs conferem a esses contaminantes a razão para a sua inclusão na maioria dos programas de monitoramento ambiental e saúde humana em diferentes países no mundo (WHO 1983, EPA 1986).

Em geral as emissões de Hidrocarbonetos Policíclicos Aromáticos no meio ambiente podem ocorrer através de três possíveis fontes: pirolítica,

Tabela I. Propriedades físico-químicas dos HPAs. Número de anéis aromáticos; $\boldsymbol{P M}$, peso molecular (g.mol ${ }^{-1}$ ); $\boldsymbol{S}$, solubilidade (mg.L ${ }^{-1}$ ); $\boldsymbol{P V}$, pressão de vapor (Pa - Pascal); $\boldsymbol{H}$, constante de Henry $\left(\mathrm{Pa} \mathrm{m}^{3} . \mathrm{mol}^{-1}\right)$; $\boldsymbol{L o g} \boldsymbol{K}_{o a}$, coeficiente de partição (octanol/água). Adaptado de Latimer \& Zheng (2003).

\begin{tabular}{|c|c|c|c|c|c|c|}
\hline HPAs & $\begin{array}{c}\mathrm{N}^{\circ} . \\
\text { de anéis }\end{array}$ & $\begin{array}{c}P M \\
(\text { g.mol-1) }\end{array}$ & $\begin{array}{c}S \\
\left(\mathrm{mg} \cdot \mathrm{L}^{-1}\right)\end{array}$ & $\begin{array}{c}P V \\
(\mathrm{~Pa})\end{array}$ & $\begin{array}{c}H \\
\left(\mathrm{~Pa} \mathrm{~m}^{3} \cdot \mathrm{mol}^{-1}\right)\end{array}$ & $\begin{array}{l}\log \\
\mathrm{K}_{\text {oa }}\end{array}$ \\
\hline Naftaleno & 2 & 128 & 31 & 10,4 & 43,01 & 3,37 \\
\hline Acenaftileno & 3 & 150 & 16,1 & 0,9 & 8,4 & 4,00 \\
\hline Acenafteno & 3 & 154 & 3,8 & 0,3 & 12,17 & 3,92 \\
\hline Fluoreno & 3 & 166 & 1,9 & 0,09 & 7,87 & 4,18 \\
\hline Fenantreno & 3 & 178 & 1,1 & 0,02 & 3,24 & 4,57 \\
\hline Antraceno & 3 & 178 & 0,045 & 0,001 & 3,96 & 4,54 \\
\hline Fluoranteno & 4 & 202 & 0,26 & 0,00123 & 1,037 & 5,22 \\
\hline Pireno & 4 & 202 & 0,132 & 0,0006 & 0,92 & 5,18 \\
\hline Benz[a]antraceno & 4 & 228 & 0,011 & $2,80.10^{-5}$ & 0,581 & 5,91 \\
\hline Criseno & 4 & 228 & nd & $5,70.10^{-7}$ & 0,065 & 5,86 \\
\hline Benz[b]fluoranteno & 5 & 252 & 0,0015 & nd & nd & 5,80 \\
\hline Benz $[\mathrm{k}]$ fluoranteno & 5 & 252 & 0,0008 & $5,20.10^{-8}$ & 0,016 & 6,00 \\
\hline Benzo[a]pireno & 5 & 252 & 0,0038 & $7,00.10^{-7}$ & 0,046 & 6,04 \\
\hline Indeno[1,2,3-cd]pireno & 6 & 278 & nd & nd & 0,003 & nd \\
\hline Dibenzo[a,h]antraceno & 5 & 278 & 0,0006 & $3,70.10^{-10}$ & nd & 6,75 \\
\hline Benzo[g,h,i]perileno & 6 & 268 & 0,00026 & nd & 0,075 & 6,50 \\
\hline
\end{tabular}

nd - Não determinado. 
petrogênica e diagênica. HPAs de origem pirolítica, resultam da combustãoincompleta dematéria orgânica sob condições de elevada temperatura, baixa pressão e curto tempo de formação. Acredita-se que esses HPAs são formados pela "quebra" ou "rompimento" da própria matéria orgânica em moléculas menores durante a pirólise, e consecutivamente rearranjados em HPAs não alquilados (Neff 1979, Meyer \& Ishiwatari 1993). Os HPAs de origem petrogênica são formados por processos diagênicos, que neste caso, remetem a condições de baixa temperatura relativa, sobre escalas geológicas de tempo. Essas condições influenciam a composição primaria de moléculas alquiladas, onde HPAs são derivados principalmente de material vegetal fóssil (Neff 1979). Processos diagênicos (qualquer mudança química, física ou biológica sofrida por um sedimento após sua deposição inicial, sob condições relativas de baixa temperatura e pressão) também podem formar HPAs através de precursores biogênicos, como por exemplo, a formação de terpenos, derivados de material vegetal e que potencialmente geram hidrocarbonetos polinucleados como perilenos, retenos e crisenos, assim como em alguns compostos derivados de fenantreno (Meyer \& Ishiwatari 1993, Silliman et al. 1998). Porém a principal via de HPAs no meio ambiente está nas atividades humanas presentes em grandes centros urbanos e complexos industriais (Pereira et al. 2002, Torres et al. 2002, Garban et al. 2002).

Uma quarta fonte potencial de HPAs está na síntese biogênica desses compostos. Acredita-se que a síntese de HPAs por microorganismos, plantas e animais seja uma fonte considerável em áreas remotas ou isoladas de fontes antropogênicas de contaminação (Krauss et al. 2005). Em especial estão as regiões tropicais, que apesar de poucos dados com relação às zonas temperadas, parecem obter misturas distintas de HPAs (Wilcke \& Amelung 2000).

Uma dessas evidências estaria na predominância de HPAs individuais como naftaleno, fenantreno e perileno, compostos esses considerados importantes indicadores da síntese natural de HPAs em regiões tropicais (Azuma et al. 1996, Wilcke et al. 2000, Krauss et al. 2005). A presença de HPAs como naftaleno e fenantreno é controversa em ambientes tropicais, tendo em vista suas características voláteis e de fácil degradação ambiental quando comparados com os HPAs de maior peso molecular. (Wilcke et al. 2000). Esse paradoxo fortalece ainda mais a hipótese de fontes intrínsecas de HPAs nessas regiões. Chen e colaboradores (1998) demonstraram que o naftaleno, por exemplo, é um importante mediador químico na comunicação entre os indivíduos da espécie de cupim Coptotermes formosanus. Wright et al. (2000), por sua vez, verificaram que a presença do naftaleno em cupinzeiros, possivelmente inibe o crescimento de determinados grupos de fungos patógenos aos mesmos (i.e. Metarhizium anisopliae).

Wilcke et al. (1999) identificaram fortes evidências de fontes biológicas na planície Amazônica. Apresença de HPAs como naftaleno, perileno e fenantreno foram detectados em tecidos de plantas, solo e nas paredes de cupinzeiros do gênero Nasutitermes sp. O naftaleno também pode ser encontrado em extratos de folha de Magnólia (Azuma et al. 1996) e em fungos endofíticos como na espécie Muscolor vitigenus, este último presente na Amazônia Peruana apresentou ação inseticida para a espécie Cephus cinctus (Daisy et al. 2002). Outros biomas como o cerrado, caatinga, pantanal e florestas de mata atlântica, também apresentam evidências de fontes naturais de HPAs. Wilcke e colaboradores (2003) verificaram uma alta freqüência relativa de fenantreno com relação aos demais HPAs em solos de florestas de mata atlântica. Isso porque o fenantreno pode ser sintetizado a partir de precursores biogênicos, como os alquil fenantrenos, encontrados principalmente em tecidos vegetais (Sims \& Overcash 1983).

Acredita-se que fontes biológicas podem estar relacionadas principalmente com a síntese de metabólitos secundários nas plantas, agindo aparentemente como uma estratégia de defesa contra a taxa de herbivoria e ações parasitóides. Contudo, tanto a avaliação de HPAs em matrizes ambientais quanto à identificação de suas origens específicas são limitadas. Isso porque muitas vezes os HPAs ocorrem no ambiente em misturas complexas (Yunker et al. 2002). Assim, a tentativa de avaliar a concentração desses contaminantes com relação ao seu perfil característico muitas vezes não é bem sucedida. $\mathrm{Na}$ maioria dos casos, a caracterização desses perfis está voltada para a identificação de HPAs com origens petrogênicas ou de combustão (Page et al. 1999, Yunker et al. 2002).

Em geral, as contribuições de HPAs de diferentes 
origens em complexas misturas caracterizam fontes não pontuais de contaminação. Já HPAs de origem petrogênica podem está associados a contaminações locais, como próximo a refinarias, rodovias e rotas marítimas de navegação (Mantis et al. 2005). HPAs petrogênicos são constituídos predominantemente por 2 e 3 anéis aromáticos (i.e. naftaleno, fluoreno e fenantreno), enquanto HPAs originários de queima apresentam uma maior freqüência relativa para compostos com 4 a 6 anéis (i.e. fluoranteno, pireno, benzo(b,k)fluorantenos e benzo(g,h,i) perileno). Vasconcellos et al. (2003), sugerem que a relativa abundância de pireno seguida de criseno e fluoranteno, observados em aerossóis da cidade de São Paulo, é originária principalmente pela exaustão de veículos motores a gasolina, enquanto criseno, pireno e benzo(a)antraceno são emitidos tanto por veículos a gasolina, como por veículos que utilizam combustível a diesel. Por outro lado, Santos et al. (2004), verificaram que a elevada freqüência relativa de benzo(a)pireno e pireno, em material particulado atmosférico, podem estar relacionadas com fontes pontuais originárias da queima de carvão por uma termoelétrica local.

Diferentes estudos vêm utilizando razões de HPAs individuais com o intuito de identificar e calcular possíveis fontes de origem desses contaminantes (Budzinski et al. 1997, Page et al. 1999, Readman et al. 2002). A utilização dessas razões está baseada na temperatura de formação dos HPAs, levando-se em conta a estabilidade química individual de cada composto. HPAs com massa moleculares de 202 (pireno e fluoranteno) e 276 (benzo[g,h,i]perileno indeno[1,2,3cd]pireno) são bons indicadores, por exemplo, na distinção entre fontes de origem petrogênicas vs. pirolíticas (Tabela II). Por outro lado, massas moleculares de 228 (i.e. criseno e benzo[a]antraceno) e 278 (i.e. dibenzo[a,h]antraceno) apresentam baixa capacidade como indicadores de origem (Brito et al. 2005). Bícego et al. (2006), ao estudar o sistema estuaríno de Santos e São Vicente (Santos, SP), verificaram uma elevada predominância deHPAs originários da combustãoporcarvão(oriundos de atividade metalúrgica), óleo cru, diesel (i.e. refinaria e navegação) e da queima de biomassa proveniente da população do entorno. Fernandes et al. (2002), também identificaram através de razões de HPAs individuais (benzo[g,h,i]perileno \ indeno[1,2,3cd] pireno) uma considerável contribuição emitida pelo tráfego automotivo. Benzo(g,h,i)perileno é reportado como um dos HPAs mais estável de sua classe. O mesmo é produzido principalmente por fontes móveis de origem, e a sua razão com indeno(1,2,3cd)pireno estima geralmente o aumento da exaustão automotora vs. possíveis fontes de origem estacionária (Oda et al. 1998).

A interação dos HPAs com outras moléculas orgânicas pode amplificar a persistência desses compostos no ambiente. No processo de pirólise, por exemplo, outros produtos como os carbonos de fuligem ("soot carbon") são produzidos, formando aglomerados de HPAs condensados. Esses produtos apresentam alta afinidade por HPAs pirolíticos e permanecem sob forma estável no ambiente,

Tabela II. Caracterização de origens de HPAs através das razões entre compostos não alquilados.

\begin{tabular}{lccl}
\hline \multicolumn{1}{c}{ Razões $^{1}$} & \multicolumn{2}{c}{ Referências } \\
& Petrogênico & Pirolítico & \\
& & & \\
Fen./Antr. & $>15$ & $<10$ & Budzinski et al. 1997 \\
Fluor./Pir. & $<1$ & $>1$ & Readman et al. 2002 \\
Naf./Fen. & $>>1$ & & Steinhauer $\&$ Boehm 1992 \\
Ind./B[ghi]P. & $>1$ & Wasserman et al. 2001 \\
Fluor./(Fluor.+ Fen.) & $<0.1$ & $>0.1$ & Yunker et al. 2002 \\
Fluor./(Fluor.+ Pir.) & $<0.5$ & $>0.5$ & Yunker et al. 2002 \\
Ind./(Ind. + B[ghi]P.) & $<0.2$ & $>0.5$ & Yunker et al. 2002 \\
\hline
\end{tabular}

${ }^{1}$ Fen. - Fenantreno; Antr. - Antraceno; Fluor. - Fluoranteno; Pir. - Pireno; Naf. - Naftaleno; Ind. - Indeno[123cd]pireno; B[ghi]P. - Benzo[ghi] perileno. 
direcionando de forma significativa processos, como a partição e a biodisponibilidade dos HPAs (Neff 1979).

Recentes estudos vêm demonstrando uma relação direta entre os níveis de HPAs no ambiente (i.e. ar, solo, sedimento e biota) com a presença humana local (Rose \& Rippey 2002, Barra et al. 2005). Hafner et al. (2005) verificaram uma estreita relação $\left(\mathrm{r}^{2}=0,92\right)$ entre a densidade populacional e a concentração de HPAs na atmosfera. Japenga et al. (1988), num estudo pioneiro de poluentes orgânicos na costa brasileira, também atribuíram a presença de HPAs no sedimento com atividades urbanas ou industriais nas proximidades dos locais de coleta.

Em geral, quando observamos níveis mundiais de HPAs na literatura internacional, podemos verificar esta relação de forma ainda mais clara. Na Tabela III, por exemplo, podemos observar os níveis de HPAs encontrados em amostras de sedimento para diferentes países do mundo. Áreas consideradas urbanas e/ou industriais apresentam níveis de HPAs superiores em até duas ordens de grandeza, quando comparadas com regiões remotas do globo, ou consideradas distantes de possíveis fontes pontuais de contaminação.

No Brasil, por exemplo, os maiores níveis de HPAs são observados nos grandes centros urbanos e industriais do país, como no caso dos estados do Rio de Janeiro e São Paulo. Nishigima et al. (2001); Bícego et al. (2006) verificaram elevadas concentrações de HPAs (80-42.390 ng.g ${ }^{-1}$ e 22,6-68.130ng.g ${ }^{-1}$ respectivamente) em sistemas estuarínos próximo ao complexo industrial de Cubatão (Santos, São Paulo). O mesmo pode ser observado nos estudos de Meniconi et al. (2002) e de Torres et al. (2002) que reportaram concentrações de 8.035ng.g ${ }^{-1}$ e 40.000ng.g ${ }^{-1}$ de HPA total em sedimento de fundo da Baía de Guanabara e do rio Paraíba do Sul (Rio de Janeiro, RJ), respectivamente. Num estudo mais recente, Pereira et al. (2007) observaram que a deposição atmosférica total de HPAs pode atingir concentrações acima de $2.000 \mathrm{ng} /\left(\mathrm{m}^{2} \mathrm{dia}\right)$ em regiões industrializadas (Volta Redonda, RJ). O mesmo estudo também utilizou biomonitores atmosféricos (Tillandsia usneoides L.) e verificou níveis de HPAs superiores em até 22 vezes aos reportados, quando comparados aos pontos controle previamente escolhidos no estudo. Entretanto, atividades agrícolas como na queima de biomassa vegetal, também são caracterizadas como fontes importantes de HPAs no ambiente (Azevedo et al. 2002, Gomes \& Azevedo 2003). Azevedo et al. 2002, observaram elevadas concentrações de HPAs no material particulado atmosférico (342ng.m ${ }^{-3}$ ) para a cidade de Campos dos Goytacazes (RJ). A cidade é caracterizada pela vasta atividade agrícola ao redor, envolvendo principalmente a monocultura de cana de açúcar.

Tabela III. Níveis de HPA total (sedimento) encontrados no mundo, de acordo com a literatura disponível.

\begin{tabular}{|c|c|c|}
\hline Localização & $\begin{array}{l}\text { HPA total }\left(\text { ng.g }{ }^{-1}\right)^{*} \\
\text { Áreas remotas }\end{array}$ & Referências \\
\hline Florida, NE (EUA) & 1.800 & Furlong et al. 1987 \\
\hline Lago larto, LA (EUA) & 100 & Catallo et al. 1995 \\
\hline Arresjoen (Ártico) & 260 & Fernández et al. 1999 \\
\hline Europa Central & 1.100 & Fernández et al. 1999 \\
\hline Lago Laja (Chile) & 359 & Quiroz et al. 2005 \\
\hline PARNA Serra da Bocaina, RJ (Brasil) & 115 & Meire 2006 \\
\hline \multicolumn{3}{|c|}{ Áreas Urbanas \& Industriais } \\
\hline Lago Michigan (EUA) & 3.500 & Simick et al. 1996 \\
\hline Lago Zurich $(\mathrm{CH})$ & 15.000 & Walkeman et al. 1980 \\
\hline Washington (EUA) & 7.000 & Walkeman et al. 1980 \\
\hline Priest pot (RU) & 16.000 & Cranwell \& Koul 1989 \\
\hline Rio Paraíba do Sul, RJ(Brasil) & 40.000 & Torres et al. 2002 \\
\hline Baía de Guanabara, RJ (Brasil) & 8.035 & Meniconi et al. 2002 \\
\hline Baía de Todos os Santos, BA (Brasil) & 4.163 & Venturini \& Tommasi 2004 \\
\hline Lagoa dos Patos, RS (Brasil) & 11.780 & Medeiros et al. 2005 \\
\hline Estuário de Santos, SP (Brasil) & 68.130 & Bícego et al. 2006 \\
\hline
\end{tabular}




\section{ASPECTOS TOXICOLÓGICOS}

HPAs são altamente lipossolúveis e rapidamente absorvidos pelos pulmões, intestinos e pela pele de animais experimentais, independente da rota de administração. O padrão de distribuição foi similar depois de administração subcutânea, intravenosa e intra traqueal, tanto em ratos quanto em camundongos. Níveis detectáveis de HPAs podem ser observados em muitos órgãos internos de minuto a horas de administração. Os maiores níveis são encontrados no fígado (IARC 1983, Forth et al. 1988). Glândula mamária e tecido adiposo, por suas características físicas e químicas, poderiam ser considerados significativos depósitos de estocagem para os HPAs (Modica et al. 1983), mas pela rápida degradação por processos metabólicos são demonstrados níveis não significativos. $\mathrm{O}$ trato gastrointestinal contém quantidades relativamente altas de metabólitos de HPAs como resultado da excreção hepatobiliar (Scheled et al. 1970). Alguns HPAs, como no caso do benzo(a)pireno, podem cruzar rapidamente a barreira placentária de ratos e camundongos (Neubert \& Tapken 1988, Withey et al. 1993) concordando com os dados sobre toxicidade para o feto e seu desenvolvimento.

O benzo(a)pireno é facilmente absorvidos no trato digestivo quando presente como soluto em vários tipos de lipídios da dieta. A absorção é facilitada na presença de sais biliares na luz intestinal. Em ratos, foi observado que entre 30 a $50 \%$ da dose oral de benzo(a) pireno e de pireno foram rapidamente absorvidas e a maior parte dos compostos foi prontamente metabolizada no fígado (efeito de primeira passagem) (Foth et al. 1988, Withey et al. 1993). HPAs como o benzo(a)pireno, fenantreno e pireno penetram rapidamente na pele de camundongos e ratos. Em camundongos, também se observou que $80 \%$ do benzo(a)pireno absorvido via dérmica foi recuperado nas fezes dos animais tratados em um período de sete dias, enquanto que em ratos a taxa de recuperação de benzo(a)pireno nas fezes e na urina foi de $42 \%$ (Sandlers et al. 1984, Yang et al. 1986).

Estudos sobre a retenção pulmonar de HPAs na forma cristalina e de HPAs em solução após instilação intra-traqueal em ratas indicaram que eles são rapidamente absorvidos pelo trato respiratório (Wolff et al. 1989). Todavia, HPAs inalados são predominantemente absorvidos sob a forma de partículas de fuligem. Após sua deposição nas vias aéreas, as partículas podem ser eliminadas por reflexo bronquial. Os HPAs podem ser parcialmente removidos das partículas por transporte na mucosa ciliada, ou podem penetrar nas células do epitélio bronquial, onde são metabolizados (WHO 1987).

Uma vez absorvido pelas células, os HPAs são metabolicamente ativados e, desta maneira, tornamse reativos a grupos nucleofílicos presentes em macromoléculas celulares. A formação de adutos de DNA é considerada essencial na carcinogenicidade química desses xenobiontes. A biotransformação dos HPAs abrange uma série de reações de oxidação, redução, hidrólise e de conjugação que são realizadas principalmente no fígado por enzimas do sistema de monooxigenases de função mista da super família dos Citocromos P450 (Klaassen et al. 1996). Este tipo de transformação tem um suposto objetivo biológico de fazer com que um determinado metabólito seja mais hidrofílico que o seu precursor, e com isso facilitar a excreção através dos fluidos biológicos. A indução de monooxigenases por substâncias químicas estranhas ao corpo é vista em diversos organismos e já foi observada em mamíferos, peixes, insetos, plantas, fungos e bactérias. Na década de 1980 já se sabia que alguns milhares de substâncias químicas naturais e sintéticas e com diversas estruturas eram capazes de aumentar os níveis de enzimas microssomais quando administradas para animais de laboratório. Drogas tais como a zoxazolamina, hormônios esteróides, alguns pesticidas e herbicidas, preservante de alimentos, certos corantes, hidrocarbonetos policíclicos aromáticos e constituintes normais da dieta são alguns exemplos de indutores de monooxigenases (Conney 1982).

O processo de oxidação enzimática seguida de hidrólise com a formação de diol-epóxidos é considerado um dos mecanismos de ativação mais aceito atualmente na literatura para bioativação dos HPAs carcinogênicos (Figura 2). A ativação desses compostos é conduzida pela enzima citocromo P450, especialmente sob duas isoformas principais: P4501A1 e P4501A2 (Hall et al. 1989). No caso do benzo(a)pireno, o citocromo P450 é responsável pelo seu metabolismo em benzo(a)pireno-7,8-óxido, que por ação subseqüente da epóxido hidrolase é transformado em benzo(a)pireno-7,8-diol. Estes dióis sofrem novamente ação do citocromo P450 e 


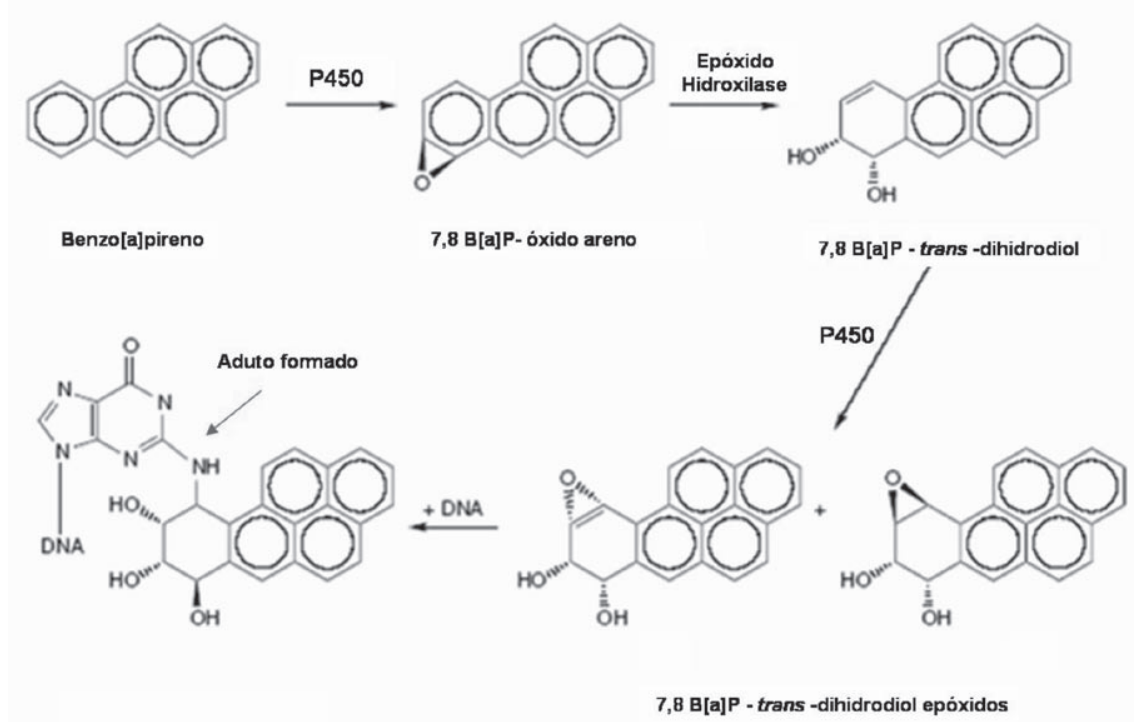

Figura 2. Esquema simplificado da ativação enzimática do benzo[a]pireno em diol epóxidos, na formação de adutos de DNA. Adaptado de Akcha et al. 2000.

transformam-se em benzo(a)pireno -7,8-diol-9,10epóxido. O benzo(a)pireno -7,8-diol-9,10-epóxido é apontado como o metabólito final de efeito carcinogênico e mutagênico, devido seu alto poder de se ligar covalentemente ao grupo 2-amino da guanina na fita dupla de DNA (Conney 1982).

A maior tendência a carcinogenicidade do benzo(a)pireno-7,8-diol-9,10-epóxido é explicada pela teoria da região de baía. Esta teoria baseou-se nas observações da notável reatividade do transbenzo(a)pireno-7,8-diol-9,10-epóxido. Esta molécula possui uma característica estrutural chamada de região de baía que é um local originário do campo de ressonância magnética onde os hidrogênios possuem ressonância menor que a do envelope aromático, por ser perdida para o próximo anel. A região de baía é um local com uma maior probabilidade de sofrer reação (Jerina et al. 1978). A elucidação de que a região de baía tinha grande importância na formação de cânceres se deu pela observação de uma marcante diminuição na formação de tumores quando eram aplicados, também em animais de laboratório, metabólitos contendo grupos alquil ou com halogênio como substituintes na região de baía (Jerina 1978).

Além do benzo(a)pireno, o benzo(b)fluoranteno, benzo(a)antraceno, indeno(1,2,3-c,d)pireno e dibenzo(a,h)antraceno são apontados como HPAs carcinogênicos em testes com animais de laboratório (Tabela IV) (Lo \& Sandi 1978, Fazio \& Howard 1983, IARC 1983, Kramers \& Van Der Heljden 1988). Estes outros HPAs carcinogênicos também sofrem modificações pelo sistema enzimático de monooxigenases até serem metatabolizados em substâncias com atividade na promoção de tumores, assim como os derivados do benzo(a)pireno. Contudo, existem outros mecanismos que também explicam a ativação de HPAs em sistemas biológicos, como a produção de radicais catiônicos, a formação de ésteres benzílicos e a produção de quinonas (Netto et al. 2000). Acredita-se que a elevada produção de adutos de DNA nas células implique em potenciais efeitos mutagênicos (Akcha et al. 2000). Um desses indícios está na mutação da proteína p53 que é responsável pelo controle da proliferação celular em mamíferos (Binková et al. 2000). Denissenko et al. (1996) demonstraram a ligação do derivado diol epóxido do benzo(a)pireno com regiões com alta freqüência de mutações no gene P53, particularmente nos códons 157, 248 e 273. O códon 157 é um local de mutação específico de tumores de pulmão não ocorrendo em outros tipos de tumores, enquanto que os outros (códon 248 e 273) são afetados em diferentes tipos de tumores (Hollstein et al. 1991, Greensblatt et al. 1994, Levine et al. 1995).

Não somente os derivados diol epóxido do benzo(a) pireno são considerados precursores de tumores. Derivados diol epóxido de outros HPAs também se caracterizam por induzir a formação de tumores em animais experimentais (WHO 1998, Ross \& Nesnow 1999, Xue \& Warsh 2005). A formação de outros metabólitos de HPAs como as ortoquinonas também 
podem levar a formação de complexos instáveis e estáveis com o DNA (Yu et al. 2007).

Tabela IV. Evidências carcinogênicas de Hidrocarbonetos Poliaromáticos em camundongos de acordo com a agência internacional de estudos sobre o câncer (WHO 1983).

\begin{tabular}{lc}
\hline HPAs & WHO \\
Fluoreno & Insuficiente \\
Fenantreno & Insuficiente \\
Antraceno & nd \\
Fluoranteno & Insuficiente \\
Pireno & nd \\
Criseno & Limitada \\
Benzo[a]Antraceno & Suficiente \\
Benzo[b]Fluoranteno & Suficiente \\
Benzo[k]Fluoranteno & Suficiente \\
Benzo[a]pireno & Suficiente \\
Dibenzo[a,h]antraceno & Suficiente \\
Benzo[g,h,i]perileno & Insuficiente \\
Indeno[1,2,3-cd]pireno & Suficiente \\
\hline & nd - Não determinado
\end{tabular}

Os primeiros casos de câncer em limpadores de chaminé expostos a produtos de combustão orgânica foram documentados em 1775 na cidade de Londres. O mesmo foi também retratado com trabalhadores alemães no início do século 19, que manipulavam carvão e piche (Eisler 1987 apud Douben 2003). Posteriormente, matrizes como carvão e óleo bruto (e seus derivados) foram testados em animais de laboratório e apresentaram efeitos cancerígenos e tumorais (Thomson \& Muller 1989). Apesar da complexidade das misturas testadas em laboratório com a presença significativa de diferentes HPAs, os estudos identificaram principalmente o benzo(a) pireno como um dos mais importantes princípios ativos cancerígenos desta classe de compostos. Em 1951, através do trabalho de Miller, evidenciouse que o benzo(a)pireno quando era metabolizado produzia compostos intermediários carcinogênicos. Outras investigações subseqüentes indicaram que a aplicação de benzo(a)pireno e outros HPAs carcinogênicos, também em pele de rato, resultava na ligação covalente dos metabólitos a proteínas, DNA e RNA. Estas transformações sofridas pelos HPAs em diversos tecidos e órgãos como pele, fígado e pulmões produzem intermediários carcinogênicos por uma cascata de reações enzimáticas (Conney 1982).
Novas técnicas para monitorar a exposição humana a carcinógenos vêm sendo desenvolvidas nos últimos anos. Os segmentos de DNA das células brancas do sangue danificados pelos HPAs e os conjugados como o benzo(a)pireno diol-epóxido excretados na urina são biomarcadores para medir a exposição aos HPAs (Strickland et al. 1996). O benzo(a)pireno diolepóxido também já foi encontrado ligado ao DNA do cólon humano e em lesões causadas pela aterosclerose (Bartsch 2000).

Hoje também se sabe que grupos de população que consomem alimentos defumados, indivíduos em contato com ambiente (água e ar) contaminado, fumantes, e pessoas ocupacionais que se expõem diretamente aos HPAs (bombeiros, trabalhadores de plantas de alumínio e de aço) eliminam produtos de degradação do benzo(a)pireno na urina (Strickland et al. 1996). A mistura de exposições e uma relativa raridade de indivíduos e grupos ocupacionais, especialmente aqueles que tenham tido contato direto com a substância em especial, são os maiores obstáculos para a avaliação epidemiológica de câncer. Um grande problema é a falta de estimativas quantitativas de exposição humana a materiais específicos (IARC 1985).

Como produtos de degradação térmica, os HPAs também vem sendo encontrados em quantidades traço na fumaça de cigarro, ar poluído e água de beber. Fumaça de tabaco está relacionada com o desenvolvimento de câncer em vários locais: pulmões, laringe, cavidade oral, esôfago, bexiga, pâncreas e rim. Na década de 1980, já se tinham evidências epidemiológicas de que ar poluído poderia contribuir para promoção de tumores, mesmo ainda não havendo dados conclusivos (IARC 1983).

Com o intuito de avaliar e monitorar possíveis grupos ocupacionais a exposição de HPAs, diferentes estudos vem utilizando medidas traço de benzo(a) pireno no ar como potenciais indicadores dos níveis de poluição atmosférica em muitos países do mundo (Fernandes et al. 2002, Santos et al. 2004). Na Figura 3 , por exemplo, podemos comparar os níveis médios de benzo(a)pireno no ar (ng. $\mathrm{m}^{-3}$ ) para diferentes metrópoles da América do Norte, do Sul, Ásia, Europa e Oceania (Fernandes et al. 2002). Para a cidade do Rio de Janeiro (0,48ng. $\left.\mathrm{m}^{-3}\right)$, por exemplo, verificamos uma semelhança nos valores de benzo(a)pireno com outros países da América do Norte e Oceania 


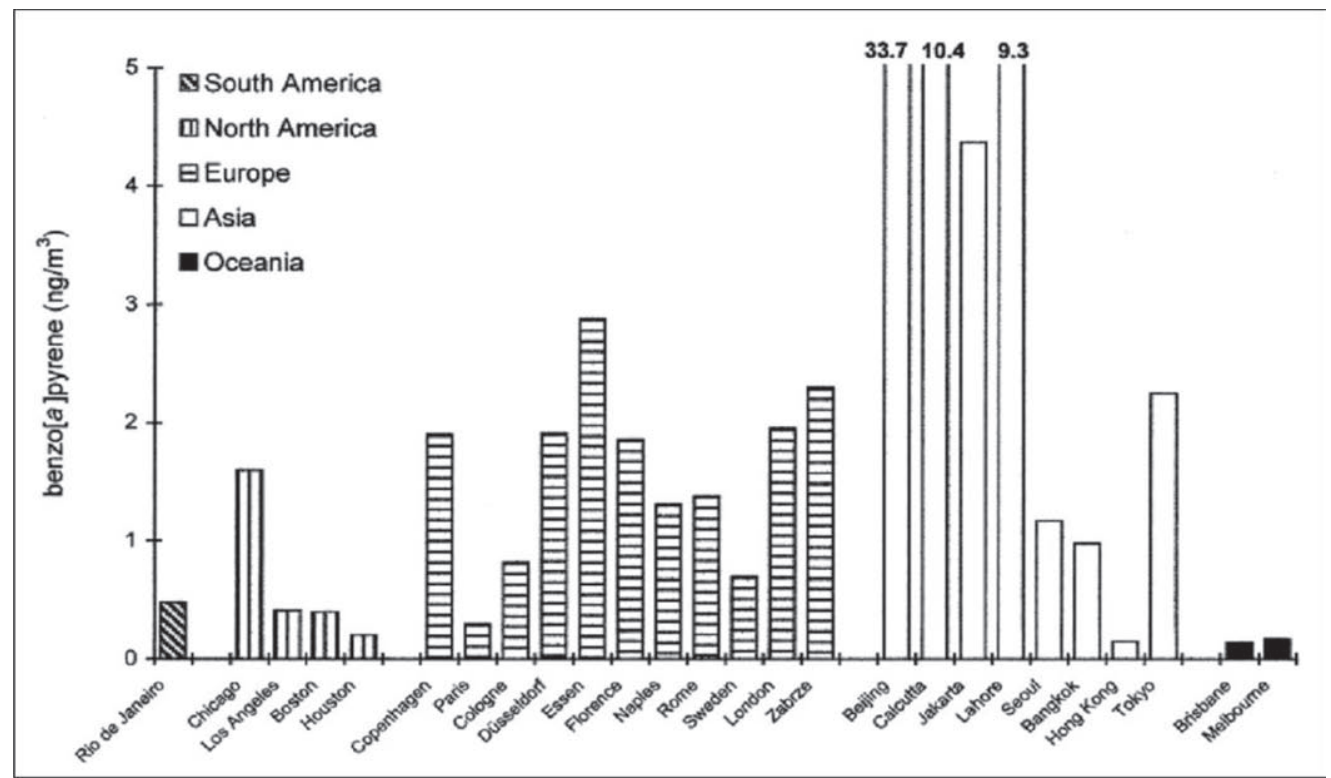

Figura 3. Níveis médios de benzo(a)pireno em ar (ng.m-3) para o Rio de Janeiro, cidades da América do Norte, Europa, Ásia e Oceania. (Fonte: Adaptado de Fernandes et al. 2002).

$\left(<2 n g \cdot m^{-3}\right)$. Concentrações um pouco mais elevadas são encontradas para alguns países da Europa (1 a 3ng. $\mathrm{m}^{-3}$ ). O mesmo pode ser observado para países desenvolvidos da Ásia como Tóquio e Hong Kong, porém valores reportados para as cidades de Beijing (China), Lahore (Paquistão) e Jaquarta (Paquistão), apresentam níveis superiores que variam de 3 a 20 vezes aos valores médios encontrados na Europa $\left(1,57 \mathrm{ng}^{-3} \mathrm{~m}^{-3}\right)$. Isso pode ser explicado pelo acelerado desenvolvimento econômico desses países, que se baseiam muitas vezes na utilização da queima de carvão como fonte principal de energia. De acordo com Fernandes et al. (2002), de 6 a 7 pessoas entre 100.000 indivíduos morrem de câncer em consequiência ao tempo de exposição a ambientes contaminados, que apresentem concentrações médias em torno de 0,75ng. $\mathrm{m}^{-3}$ de benzo(a)pireno no ar (Harrinson 1998). Isso pode representar uma estimativa em torno de 720 casos extra de câncer numa população exposta de aproximadamente 11.000 .000 de pessoas.

AGRADECIMENTOS: Ao fomento da CAPES, FAPERJ, CNPqPROSUL (edital: 016/2004) \& Sinai Medicine Mount of School (No.1 D43 TW00640).

\section{REFERÊNCIAS}

AKCHA, F.; IZUEL, C.; BUDZINSKI, H.; BURGEOT, T. \& NARBONNE, J.F. 2000. Enzymatic biomarker measurement and study of DNA adduct formation in $\mathrm{B}[\mathrm{a}] \mathrm{P}$-contaminated mussel, Mytilus galloprovincialis. Aquatic Toxicology, 49: 269-287.

AZEVEDO, D.A.; SANTOS, C.Y.M. \& NETO, F.R.A. 2002. Identification and seasonal variation of atmospheric organic pollutants in Campo dos Goytacazes, Brazil. Atmospheric Environment, 36: 2383-2395.

AZUMA, M.; TOYOTA, Y.A. \& KAWATO, S. 1996. Naphthalene - a constituent of Magnólia flowers. Phytochemistry, 42: 999-1004.

BARRA, R.; POPP, P.; QUIROZ, R.; BAUER, C.; CID, H. \& TUMPLING, W.V. 2005. Persistent toxic substances in soils and waters along an altitudinal gradient in the Laja river basin, central southern Chile. Chemosphere, 58: 905-915.

BÍCEGO, C.M.; TANIGUCHI, S.; YOGUI, G.T.; MONTONE, R.C.; SILVA, D.A.M.; LOURENÇO, R.A.; MARTINS, C.C.; SASAKI, S.T.; PELLIZARI, V.H. \& WEBER, R.R. 2006. Assessment of contamination by polychlorinated biphenyls and aliphatic and aromatic hydrocarbons in sediments of the Santos and São Vicente Estuary System, São Paulo, Brazil. Marine Pollution Bulletin. 52(12): 1804-1816.

BINKOVÁ, B.; GIGUÈRE, Y.; RÖSSNER-JR, P. DOSTÁL, M. \& SRÁM. R. 2000. The effect of dibenzo[a,h]pyrene and benzo[a]pyrene on human diploid lung fibroblasts: the induction of DNA adducts, expression of p53 and p21 $1^{\text {WAF1 }}$ proteins and cell cycle distribution. Mutation Research/ Genetic Toxicology and Environmental Mutagenesis, 471(1-2): 57-70.

BRITO, E.M.S.; VIEIRA; E.D.R.; TORRES, J.P.M. \& MALM, 
O. 2005. Persistent organic pollutants in two reservoirs along the Paraíba do sul-Guandu river system, Rio de Janeiro, Brazil, Química Nova, 28(6): 941-942.

BRITO, E.M.S; GUYONEAUD, R.; GOÑI-URRIZA, M.; RANCHOU-PEYRUSE, A.; VERBAERE, A.; CRAPEZ, M.A.C.; WASSERMAN J.C.A. \& DURAN, R. 2006. Characterization of hydrocarbonoclastic bacterial communities from mangrove sediments in Guanabara Bay, Brazil. Research in Microbiology, 15(8): 752-762.

BUDZINSKI, H.; JONES, I.; BELLOCQ, J.; PIÉRARD, C. \& GARRINGUES, P. 1997. Evaluation of sediment contamination by policyclic aromatic hydrocatbons in the Gironde estuary. Marine Chemistry, 58: 85-97.

CATALLO, W.J.; SCHLENKER, M.; GAMBRELL, R.P. \& SHANE, B.S. 1995. Toxic chemicals and trace metals from urban and rural Louisiana lakes: recent historical profiles and toxicological significance. Environmental Science \& Technology, 29: 1436-1445.

CHAILLAN, F.; LE FLÈCHE, A.; BURY, E.; PHANTAVONG, Y.; GRIMONT, P.; SALIOT, A. \& OUDOT, J.; 2004. Identification and biodegradation potential of tropical aerobic hydrocarbon-degrading microorganisms. Research in Microbiology, 155: 587-595.

CHEN. J.; HENDERSON, G.; GRIMM, C.C.; LLOYD, S.W. \& LAINE, R.A. 1998. Termites formigate their nest with naphthalene. Nature, 392: 558.

CONNEY, A.H. 1982. Introduction of microssomal enzymes by foreign chemicals and carcinogenesis by polycyclic aromatic hydrocarbons: G. H. A. Clowes memorial lecture. Cancer Research, 42: 4875-4917.

CRANWELL, P.A.\& KOUL, V.K. 1989. Sedimentary record of Polycyclic Aromatic and Aliphatic Hydrocarbons in the Windermere catchment. Water research, 23: 275-283.

DAISY, H.B.; STROBEL, G.A.; CASTILLO, U.; EZRA, D.; SEARS, J.; WEAVER, D.K. \& RUNYON, J.B. 2002. Naphthalene, an insect repellent, is produced by Muscodor vitigenus, a novel endophytic fungus. Microbiology, 148: 3737-3741.

DENISSENKO, M.F.; PAO, A.; TANG, M. \& PFEIFER, G.P. 1996. Preferencial formation of benzo(a)pyrene adducts at lung cancer mutational hotspots in P53. Science, 247: 430-432.

DOUBEN, P.E.T. 2003. PAHs: An Ecotoxicological perspective (first edition). John Wiley \& Sons, Ltda. England. ISBN 0-471-56024-3. Pp 389.

ENVIRONMENT PROTECTION AGENCY - EPA 1986. Quality criteria for water 1986. EPA 440/5-86-001. US Environmental Protection Agency, Washington, DC.
FAZIO, T. \& HOWARD, J.W. 1993. Polycyclic aromatic hydrocarbons in foods. In: Handbook of Polycyclic Aromatic Hydrocarbons, ed. Bjørseth, A., Marcel Dekker, New York, 1983, vol.1. Pp 461.

FERNANDES, M.B; BRICKUS, L.S.R; MOREIRA, J.C \& CARDOSO, J.N. 2002. Atmospheric BTX and polyaromatic hydrocarbons in Rio de Janeiro, Brazil. Chemosphere, 47: 417-425.

FERNÁNDEZ, P.; VILANOVA, R.M. \& GRIMALT, J.O. 1999. Sediment fluxes of Polycyclic Aromatic Hydrocarbons in European high altitude mountain lakes. Environmental Science \& Technology, 33: 3716-3722.

FOTH, H.; KAHL, R. \& KAHL, G.F. 1988. Pharmacokinetics of low doses of benzo[a]pyrene in the rat. Food Chemistry \& Toxicology, 26: 45-51.

FURLONG, E.T.; CESSAR, L.R. \& HITES, R.A. 1987. Accumulation of polycyclic aromatic hydrocarbons in acid sensitive lakes. Geochimica et Cosmochimica Acta, 51: 2965-2975.

GARBAN, B.; BLANCHOUD, H.; MOTALAY-MASSEI, A.; CHEVREUIL, M. \& OLLIVON, D. 2002. Atmospheric bulk deposition of PAHs onto France: trends from urban to remote sites. Atmospheric Environment, 36: 5395-5403.

GOMES, A.O. \& AZEVEDO, D.A. 2003. Aliphatic and aromatic hydrocarbons in tropical recent sediments of Campo dos Goytacazes, RJ, Brazil. Journal Brazillian Chemistry Society, 14(3): 358-368.

GREENBLATT, M.S.; BENNETT, W.P.; HOLLSTEIN, M. \& HARRIS. C.C. 1994. Mutations in the p53 Tumor Suppressor Gene: Clues to Cancer Etiology and Molecular Pathogenesis. Cancer Research 54 : 4855-4878.

HAFNER, W. D.; CARLSON, D.L.\& R. A. HITES. 2005. Influence of local human population on atmospheric polycyclic aromatic hydrocarbons concentrations. Environmental Science \& Technology, 39(19): 7374 -7379.

HALL, M.; FORRESTER, L.M.; PARKER, D.K.; GROVER, P.L. \& WOLF, C.R. 1989. Relative contribution of various forms of cytochrome P450 to the metabolism of benzo[a]pyrene by human liver microsomes. Carcinogenesis, 10: 1815-1821.

HARRISON, R.M., 1998. Setting health-based air quality standards. Pp 57-73 In: Hester, R.E., Harrison, R.M. (Eds.), Air Pollution and Health, Issues in Environmental Science and Technology 10. The Royal Society of Chemistry, Cambridge, UK.

HEDLUND, B.P; GEISELBRECHT, A.D. \& STALEY, J.T; 2001. Marinobacter strain NCE312 has a Pseudomonas-like naphthalene dioxygenase. FEMS Microbiology Letter, 201: 47-51. 
HOLLSTEIN, M.; SIDRANSKY, D.; VOGELSTEIN, B. \& HARRIS. C.C. 1991. P53 mutations in human cancers Science, 253: 49-53.

HWANG, S. \& CUTRIGHT, T.J. 2002. Biodegradability of aged pyrene and phenanthrene in a natural soil. Chemosphere, 47 : 891-899.

IARC - INTERNATIONAL AGENCY FOR RESEARCH ON CANCER. Monographs on the Evaluation of the Carcinogenic Risk of Chemical to Humans. Polycyclic Aromatic Compounds. Part 1, Chemical, Environmental and Experimental Data, v.32. Lyon, France.

IARC - INTERNATIONAL AGENCY FOR RESEARCH ON CANCER 1985. Monographs on the Evaluation of the Carcinogenic Risk of Chemical to Humans, v.35. Lyon, France.

JAPENGA， J.; WAGENAAR, W.J.; SALOMONS, W.; LACERDA, L.D.; PATCHINEELAM, S.R.\& LEITÃOFILHO, C.M. 1988. Organic micropollutants in the Rio de Janeiro coastal region, Brazil. The Science of the Total Environment, 75: 249-259.

JERINA, D.M.; THAKKER, D.R. \& YAGI, H. 1978. Carcinogenicity of benzo(a)pyrene derivates: the bay region theory. Pure and Applied Chemistry, 50: 1033-1044.

KLAASSEN, C.D.; DOULL, J. \& AMDUR, M.O. 1996. Casarett and Doull's Toxicology - the basic science of poisons. (5 $5^{\text {th }}$ Edition). McGraw-Hill. New York. ISBN0-07-105476-6.

KRAUSS, M.; WILCKE, W.; CHRISTOPHER, M.; ADELMAR, G. B.; MARCOS, V.B.G. \& WULF, A. 2005. Atmospheric versus biological sources of polycyclic aromatic hydrocarbons (PAHs) in a tropical rain forest environment. Environmental Pollution, 135: 143-154.

KRAMERS, P.N.G. \& VAN DER HELIDEN, C.A. 1988. Polycyclic aromatic hydrocarbons (PAH): carcinogenicity data and risk extrapolation. Toxicological and Environmental Chemistry, 16: 41-351.

LATIMER, J.S. \& ZHENG, J. 2003. The sources, transport, and fate of PAHs in the marine environment. In: PAHs: An ecotoxicological perspective. John Wiley \& Sons, Ltd. ISBN: 0-471-56024-3. Pp 9-33 389.

LEVINE, A.J.; WU, M.C.; CHANG, A.; SILVER, A.; ATTIYEH, E.F.; LIN, J. \& EPSTEIN, C.B. 1995. The spectrum of mutations at the p53 locus. Evidence for tissue-specific mutagenesis, selection of mutant alleles, and a "gain of function" phenotype. Annals of the New York Academy of Science, 768: 111-128.

LO, M.T. \& SANDI, E. 1978. Polycyclic aromatic hydrocarbons (polynuclears) in foods. Residue Reviews, 69: 35-86.

MANTIS, J.; CHALOULAKOU, A. \& SAMARA, C. 2005.
PM10-bound polycyclic hydrocarbons (PAHs) in the greater area of Athens, Greece. Chemosphere, 59: 593-604.

MEDEIROS, P.M.; BÍCEGO, M.C.; CASTELAO, R.M.; ROSSO, C.D.; FILLMANN, G. \& ZAMBONI, A.J. 2005. Natural and anthropogenic hydrocarbon inputs to sediments of Patos Lagoon Estuary, Brazil. Environment International, 31: $77-87$

MEIRE, R.O. 2006. Avaliação de hidrocarbonetos policíclicos aromáticos (HPAs) em áreas de proteção permanente sudeste brasileiro. Dissertação de Mestrado, Universidade Federal do Rio de Janeiro, Instituto de Biofísica Carlos Chagas Filho. Pp 53.

MENICONI, M.F.G.; GABARDO, I.T.; CARNEIRO, M.E.R.; BARBANTI, S.M.; SILVA, G.C. \& MASSONE, C.G. 2002. Brazilian Oil Spills Chemical Characterization-Case Studies. Environmental Forensics, 3(3-4): 303-321

MEYER, P.A. \& ISHIWATARI, R. 1993. Lacustrine organic geochemistry - an overview of indicators of organic matter soucers and diagenesis in lake sediments. Organic Geochemistry, 20: 867-900.

MODICA, R.; FIUME, M.; GUAITANI, A. \& BARTOSEK, I. 1983. Comparative kinetics of benz(a)anthracene, chrysene and triphenylene in rats after oral administration. I. Study with single compounds. Toxicology Letters, 18: 103-109.

NEFF, J.M. 1979. Polycyclic Aromatic Hydrocarbons in the Aquatic Environment Sources, fate, and biological effects. Applied Science Publishers, London.

NEFF, J.M. 1984. Bioacumulation of organic micropollutants from sediments and suspended particulates by aquatic animals. Fresenius Journal of Analytical Chemistry, 319: 132-136.

NETTO, A.D.P.; DIAS, J.C.M.; ARBILLA, G.; OLIVEIRA, L.F. \& BAREK, J. 2000. Avaliação da contaminação humana por Hidrocarbonetos Policíclicos Aromáticos e seus derivados nitratos: Uma revisão metodológica, Química Nova, 23(6): 765-773.

NEUBERT, D. \& TAPKEN, S.; 1988. Transfer of benzo(a)pyrene into mouse embryos and fetuses. Archives of Toxicology, 62: 236-239.

NISHIGIMA, F.N.; WEBER, R.R. \& BICEGO, M.C., 2001. Aliphatic and aromatic hydrocarbons in sediments of Santos and Cananeia, SP, Brazil. Marine Pollution Bulletin, 42: 1064-1072.

ODA, J.; MAEDA, I.; MORI, T.; YASUHARA, A. \& SAITO, Y., 1998. The relative proportions of polycyclic aromatic hydrocarbons and oxygenated derivatives in accumulated organic particulates as affected by air pollution sources. Environmental Technology, 19: 961-976.

PAGE, D.S.; BOEHM, P.D.; DOUGLAS, G.S.; BENCE, A.E.; 
BURNS, W.A. \& MANKIEWICZ, P.J. 1999. Pyrogenic Polycyclic Aromatic Hydrocarbons in sediments record past human activity: A case study in Prince William Sound, Alaska. Marine Pollution Bulletin, 38: 247-266.

PEREIRA, P.A.P.; ANDRADE, J.B. \& MIGUEL, A.H. 2002. Measurements of semivolatile and particulate polycyclic aromatic hydrocarbons in a bus station and an urban tunnel in Salvador, Brazil. Journal of Environmental Monitoring, 4: 558-561.

PEREIRA S.M.; HEITMANN, D.; REIFENHÄUSER, W.; MEIRE, R.O.; SANTOS L.S.; TORRES J.P.M.; MALM, O. \& KÖRNER, W. 2007. Persistent organic pollutants in atmospheric deposition and biomonitoring with Tillandsia usneoides ( $L$.) in an industrialized area in Rio de Janeiro state, southeast Brazil - Part II: PCB and PAH. Chemosphere, 67: 1736-1745.

QUIROZ, R.; POPP, P.; URRUTIA, R.; BAUTER, C.; ARANEDA, A.; TREUTLER, H.C. \& BARRA, R. 2005. PAH fluxes in the Laja lake os south central Chile Andes over the last 50 years: Evidence from a dated sediment core. Science of the Total Environment, 349(1-3): 150-160.

READMAN, J.W.; FILLMANN, G.; TOLOSA, I.; BARTOCCI, J.; VILLENEUVE, J.P.; CATINNI, C. \& MEE, L.D. 2002. Petroleum and PAH contamination of the Black Sea. Marine Pollution Bulletin, 44: 48-62.

ROSE, N.L. \& RIPPEY, B. 2002. The historical record of PAH, $\mathrm{PCB}$, trace metal and fly-ash particle deposition at a remote lake in noth-west Scotland. Environmental pollution, 117: 121-132.

ROSS J.A. \& NESNOW S. 1999. Polycyclic aromatic hydrocarbons: correlations between DNA adducts and ras oncogene mutations. Mutation Research, 424: 155-66.

SANDERS,C.L.; SKINNER, C. \& GELMAN, R.A. 1984. Percutaneous absorption of benzo[a]pyrene and dimethylbenz[a]anthracene in mice. Environmental Research, 33: 353-360.

SANTOS, C.Y.M.; AZEVEDO, D.A.A. \& NETO, F.R.A. 2004. Atmospheric distribution of organic compounds from urban areas near a coal-fired power station. Atmospheric Environment, 38: 1247-1257.

SCHLEDE, E.; KUNTZMAN, R. \& CONNEY, A.H. 1970. Stimulatory effect of benzo(alpha)pyrene and phenobarbital pretreatment on the biliary excretion of benzo(alpha)pyrene metabolites in the rat. Cancer Research, 30: 2898-2904.

SILLIMAN J.E.; MEYERS P.A. \& EADIE, B.J. 1998. Perylene: an indicator of alteration processesor precursor materials ? Organic Geochemistry, 29: 1737-1744.

SISINNO, C.L.S.; NETTO, A.D.P.; REGO, E.C.P. \& LIMA, G.S.
2003. Hidrocarbonetos policíclicos Aromáticos em resíduos sólidos industriais: uma avaliação preliminar do risco potencial de contaminação ambiental e humana em áreas de disposição de resíduos. Caderno de Saúde Pública, 19(2): 671-676.

SIMICK, M.; EISENREICH, S.; GOLDEN, K.; LIU, S.; LIPIATOU, E. \& SWACKHAMER, D.; 1996. Atmospheric loading of Polycyclic Aromatic Hydrocarbons to Lake Michigan as recorded in the sediments. Environmental Science and Technology, 30: 3039-3046.

SIMS, R.C. \& OVERCASH, M.R. 1983. Fate of polynuclear aromatic compounds in soil plant systems. Residue Reviews, 88: 1-68.

STEINHAUER, MS; BOEHM, PD. 1992. The Composition and Distribution of Saturated and Aromatic Hydrocarbons in Nearshore Sediments, River Sediments, and Coastal Peat of the Alaskan Beaufort Sea: Implications for Detecting Anthropogenic Hydrocarbon Inputs. Marine Environmental Research, 33(4): 223-253.

STRICKLAND, P.; KANG, D. \& SITHISARANKUL, P. 1996. Polynuclear aromatic hydrocarbons metabolites in urine as biomarkers of exposure and effect. Environmental Health Perspectives, 104: 927-932.

THOMSON, B. \& MULLER, P. 1989. Approaches to the estimation of cancer risk from ingested PAH. Polycyclic Aromatic Compounds, 12: 249-260.

TORRES, J.P.M.; MALM, O.; VIEIRA, E.D.R.; JAPENGA, J. \& KOOPMANS, G.F. 2002. Organic micropollutants on river sediments from Rio de Janeiro, Southeast Brazil, Caderno de Saúde Pública, 18 (2): 477-488.

VASCONCELLOS, P.C.; ZACARIAS, D.; PIRES, M.A.F.; POOL, C.S. \& CARVALHO, L.R.F. 2003. Measurements of polycyclic aromatic hydrocarbons in airborne particles from the metropolitan area of São Paulo City, Brazil. Atmospheric Environment, 37(21): 3009-3018.

VENTURINI, N. \& TOMMASI, L.R. 2004. Polycyclic aromatic hydrocarbons and changes in the trophic structure of polychaete assemblages in sediments of Todos os Santos Bay, Northeastern, Brazil. Marine Pollution Bulletin, 48: 97-107. WALKEMAN, S.; ACHAFFNER, C. \& GLGER, G. 1980. Polycyclic Aromatic Hydrocarbons in recent lake sediments: II. Compounds derived from biogenic precursors during early diagenesis. Geochimica et Cosmochimica Acta, 44: 415-429.

WARSHAWSKY, D. 1999. Polycyclic aromatic hydrocarbons in carcinogenesis. Environmental Health Perspectives. 107: 317-319.

WASSERMAN, J.C.; FIGUEIREDO, A.M.G.; PELLEGATTI, F. \& SILVA-FILHO, E.V. 2001. Elemental composition of 
sediment cores from a mangrove environment using neutron activation analysis. Journal of Geochemical Exploration, 72(2): 129-146.

WHO - WORLD HEALTH ORGANIZATION. 1983. Evaluation of the carcinogenic risk of chemicals to humans, Polynuclear Aromatic Compounds, Part 1, chemical environmental and experimental data, 32. International Agency for Research on Cancer, World Health Organization. Pp 477.

WHO - WORLD HEALTH ORGANIZATION. 1987. Polynuclear aromatic hydrocarbons $(\mathrm{PAH})$. In: Air quality guidelines for Europe. Copenhagen, World Health Organization Regional Office for Europe.

WHO - WORLD HEALTH ORGANIZATION. 1998. IPCS Environmental Health Criteria 202. Selected non-heterocyclic polycyclic aromatic hydrocarbons. Geneva: World Health Organization.

WIERSMA, D.A. \& ROTH, R.A. 1983. Total body clearance of circulating benzo(a)pyrene in conscious rats: effect of pretreatment with 3-methylcholanthrene and the role of liver and lung. Journal of Pharmacology and Experimental Therapy, 226: 661-667.

WILCKE, W.; LILIENFEIN, J.; LIMA, S.D.C. \& ZECH, W. 1999. Contamination of highly weathered urban soils in Uberlândia, Brazil. Journal of Soil Science and Plant Nutrition, 162: 539548.

WILCKE, W. \& AMELUNG, W. 2000. Persistent organic pollutants in native grassland soils along a climosequence in North America. Soil Science Society of America Journal, 64: 2140-2148

WILCKE, W.; AMELUNG, W.; MARTIUS, C.; GARCIA, M.V.B. \& ZECH, W. 2000. Biological sources of polycyclic aromatic hydrocarbons (PAHs) in the Amazonia forest. Journal of Soil Science and Plant Nutrition, 163: 27-30.

WILCKE, W.; AMELUNG, W.: KRAUSS, M.; MARTIUS, C.; BANDEIRA, A. \& GARCIA, M. 2003. Polycyclic Aromatic Hydrocarbons (PAH) patterns in climatically different ecological zones of Brazil. Organic Chemistry, 34: $1407-1417$

WITHEY, J.R.; SHEDDEN, J.; LAW, F.C. \& ABEDINI, S. 1993. Distribution of benzo[a]pyrene in pregnant rats following inhalation exposure and a comparison with similar data obtained with pyrene. Journal of Applied Toxicology, 13: 193-202.

RIGHT, M.S.; LAX, A.R.; HENDERSON, G.; CHEN, J. 2000. Growth response of Metarhizium anisopliae to two Formosan subterranean termite nest volatiles, naphthalene and fenchone. Mycologia, 92: 42-45.

WOLFF, R.K.; GRIFFITH, W.C.; HENDERSON, R.F.; HAHN,
F.F.; HARKEMA, J.R.; REBAR, A.H.; EIDSON, A.F. \& McCLELLAN, R.O. 1989. Effects of repeated inhalation exposures to 1-nitropyrene, benzo[a]pyrene, Ga2O3, particles, and SO3 alone and combinations on particle clearance, bronchoaveolar lavage fluid composition, and histopathology. Journal of Toxicology and Environmental Heath, 27: 123-138.

XUE, W. \& WARSHAWSKY, D. 2005. Metabolic activation of polycyclic and heterocyclic aromatic hydrocarbons and DNA damage: a review. Toxicology and Applied Pharmacology, 206: 73-93.

YANG, J.J.; ROY, T.A. \& MACKERER, C.R. 1986. Percutaneous absorption of benzo[a]pyrene in the rat: comparison of in vivo and in vitro results. Toxicology and Industrial Health, 2: 409-416.

YU, Z.; PALKOVICOVA, L.; DROBNA, B.; PETRIK, J.; KOCAN, A.; TRONOVEC, T. \& HERTZ-PICCIOTTO, I. 2007. Comparison of organochlorine concentrations in colostrums and mature milk. Chemosphere, 66: 1012-1018.

YUNKER, M. B.; MACDONALD, R.W.; VINGARZAN, R.; MITCHELL, H.; GOYETTE, D. \& SYLVESTRE, S. 2002. PAHs in the Fraser river basin: a critical appraisal of PAH rations as indicators of PAH source and composition. Organic Geochemistry, 33: 489-515. 